\title{
Evaluation of the chemical interactions in co-culture elements of Castanea sativa Miller mycorrhization
}

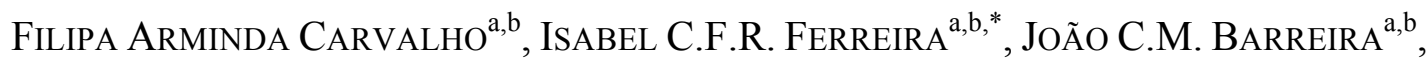
LILLIAN BARROS ${ }^{\mathrm{a}, \mathrm{b}}$, ANABELA MARTINS, $^{\mathrm{b}}$

${ }^{a}$ CIMO-ESA, Instituto Politécnico de Bragança, Campus de Santa Apolónia, Apartado 1172, 5301-855 Bragança, Portugal.

${ }^{\mathrm{b}}$ Escola Superior Agrária, Instituto Politécnico de Bragança, Campus de Santa Apolónia, Apartado 1172, 5301-855 Bragança, Portugal.

* Author to whom correspondence should be addressed (e-mail: iferreira@ipb.pt telephone +351-273-303219; fax +351-273-325405). 


\begin{abstract}
In the early steps of mycorrhizal associations an oxidative burst might occur through the rapid production of high amounts of reactive oxygen species in response to external stimuli, increasing the production of antioxidants in plant and/or mycelia. Herein, the effect of mycorrhizal association among Pisolithus arhizus or Paxillus involutus and Castanea sativa Miller (stems and roots) was studied for different co-culture periods, defined for a better comprehension of the chemical interactions in the early stages of mycorrhization $(6,24$ and $72 \mathrm{~h})$. Knowledge on the specificities of plant-host interaction provided information about the most suitable fungus to be included in the development of new management strategies, and the fungal species that induced the strongest response with increasing antioxidant activity (free radical scavenging activity, reducing power and lipid peroxidation inhibition) and production of antioxidant compounds (phenolics and tocopherols) and sugars. P. involutus seemed to be the most adequate fungus to mycorrhize with $C$. sativa. Considering bioactive compounds production, $P$. arhizus was more efficient since allowed an increase in the contents of sugars and tocopherols in all co-culture elements.
\end{abstract}

Keywords: Ectomycorrhizal fungi; Castanea sativa stems/roots; co-culture period; oxidative stress 


\section{Introduction}

Chestnut tree (Castanea sativa Miller) is cultivated all over Portugal, but with high predominance in the coldest areas in the north. Trás-os-Montes region comprises 16352 orchards $(75.8 \%$ of Portuguese production) corresponding to 23338 ha $(84.9 \%$ of Portuguese area) (Agricultural Statistics, 2010). In addition to its importance as a nut (chestnuts constitute the most important export product of the Portuguese fruit sector) and timber (chestnut wood is one of the preferred choices in making high quality furniture) producer, chestnut tree might represent another important income associated with the collection and commercialization of wild edible mushrooms growing in chestnut stands. Despite the poor knowledge of their biodiversity, ecology or sustainable management in those regions, mushroom harvesting is increasing, mainly due to their economic importance to local populations (Baptista et al., 2010). Among the ectomycorrhizal (ECM) fungi known for having positive effects on plant hosts, e.g., Pisolithus arhizus (Martins, 1997; Martins et al., 1997), Laccaria laccata, Hebeloma crustuliniforme, H. sinapizans and Paxillus involutus (Branzanti et al., 1999), a special dedication has been devoted to P. arhizus and P. involutus (Carocho et al., 2012; Reis et al., 2011; Reis et al., 2012). Our research group conducted a few studies on symbiotic associations using Pinus pinaster as the plant host (Carocho et al., 2012; Reis et al., 2011; Reis et al., 2012). Regarding this Pinaceae family plant, P. arhizus and $P$. involutus induced different responses in the plant root chemical composition and antioxidants production, revealing singular compatibilities: $P$. arhizus seemed to be more compatible than $P$. involutus, since its antioxidant activity did not show variations (Carocho et al., 2012; Reis et al., 2012). However, these ECM fungi can also associate with other tree species with high production areas like $C$. sativa, which has been studied 
by us, regarding the antioxidant activity of its flowers (Barreira et al., 2008; Barros et al., 2010), leaves, skins and fruits (Barreira et al., 2008).

Due to the importance of this species in Trás-os-Montes region, it is fundamental to improve the knowledge of ECM dynamic interactions allowing sustained decisions about crop management. The metabolic adjustments induced by mycorrhization are often related with a physiological response to a different oxidative environment, which influences the antioxidant potential of each associated element (plant constituent, mycelium or culture media). In the early steps of mycorrhizal associations an oxidative burst might occur through the rapid production of high amounts of ROS (reactive oxygen species) in response to external stimuli (Lamb and Dixon, 1997; Baptista et al., 2007). Therefore, the production and/or activity of antioxidants, including enzymes (Münzenberger et al., 1997; Baptista et al., 2007) or phenolic compounds (Münzenberger et al., 1995; Reis et al., 2011), might increase in plant roots and/or mycelia.

Attending to the mycorrhizal association and plant physiology it would be expected that roots and stems were the primary organs affected by ECM fungi. Therefore, the effect of mycorrhizal association among $P$. arhizus or $P$. involutus and $C$. sativa (stems and roots) was studied for different co-culture periods that were defined for a better comprehension of the chemical interactions in the early stages of mycorrhization. Knowledge on the specificities of plant-host interaction could provide information about: i) the most suitable fungus to be included in the development of new management strategies; or ii) the fungal species that induces the strongest response, increasing its interest as a new natural source of bioactive compounds. 


\section{Materials and methods}

\subsection{In vitro production of mycelia and germination of Castanea sativa seeds}

Mycelia of Paxillus involutus (Batsch) Fr. and Pisolithus arhizus (Scop.) Rauschert were isolated from sporocarps (Figure 1A,B; collected in Bragança, Portugal) on solid Melin-Norkans medium (MMN) pH $6.6\left(\mathrm{NaCl} 0.025 \mathrm{~g} / \mathrm{l} ;\left(\mathrm{NH}_{4}\right)_{2} \mathrm{HPO}_{4} 0.25 \mathrm{~g} / 1 ; \mathrm{KH}_{2} \mathrm{PO}_{4}\right.$ $0.50 \mathrm{~g} / \mathrm{l} ; \mathrm{FeCl}_{3} 0.0050 \mathrm{~g} / \mathrm{l} ; \mathrm{CaCl}_{2} 0.050 \mathrm{~g} / \mathrm{l} ; \mathrm{MgSO}_{4} .7 \mathrm{H}_{2} \mathrm{O} 0.15 \mathrm{~g} / \mathrm{l}$; thiamine $0.10 \mathrm{~g} / \mathrm{l}$ glucose $10 \mathrm{~g} / \mathrm{l}$; casamino acids $1.00 \mathrm{~g} / \mathrm{l}$, malt $5.00 \mathrm{~g} / 1$ and agar $20 \mathrm{~g} / 1$ in tap water) (Marx, 1969). The strains were maintained in Petri dishes (9 $\mathrm{cm}$ diameter) containing the same medium at $25^{\circ} \mathrm{C}$ in the dark and subcultured every 4 weeks (Figure 1C,D). Both mycelium and culture medium were weighted separately to obtain the fresh biomass (fw).

Castanea sativa (Mill.) seeds (locally obtained Portugal) were sterilized with sodium hypochloride $5 \%$ and two drops of tween ${ }^{\circledR} 80$ under agitation for 15 min (Harvengt, 2005). After washes with sterilized water, the seeds were poured in ethanol $80 \%$ for 15 min, washed again with sterilized water, and finally seeded in flasks with sterile wet sand o induce germination, the flasks were kept in the dark at $25^{\circ} \mathrm{C}$ for $48 \mathrm{~h}$, and then exposed to light for day and night photoperiods $(16 \mathrm{~h} / 8 \mathrm{~h})$, respectively, in a culture chamber (Gro-Lux, Sylvania) with Daylight lamps (Phillips, Amsterdam, Netherlands) (Figure 1E,F).

\subsection{Induction of the mycorrhizal symbiosis}

Fungi mycelium was cultured in Petri dishes (12 cm diameter) with incomplete MMN (without malt and casamino acids) in slant and maintained for 20 days in the dark. One C. sativa seedling was introduced in each Petri dish over the mycelium, and left in coculture in the culture chamber and conditions mentioned above (Figure 1G,H). The part 
of the Petri dish where the plant root were in contact with fungi was protect from light with aluminium paper, in order to mimetize the natural darkconditions of mycorrhizas development. After 0, 6, 24 and $72 \mathrm{~h}$ of growth, mycelium and plant root were recovered from the medium. Mycelium, plant and culture medium were weighted separately to obtain the fresh biomass (fw), and then stored at $-40^{\circ} \mathrm{C}$ for further analyses. All the samples were lyophilised (FreeZone 4.5 model 7750031, Labconco, Kansas, USA) to obtain the dry biomass ( $\mathrm{dw}$ ) and further reduced to a fine dried powder (20 mesh).

\subsection{Standards and reagents}

Acetonitrile $99.9 \%, n$-hexane $95 \%$, ethyl acetate $99.8 \%$ and methanol were of HPLC grade from Fisher Scientific (Loures, Portugal). The standards sugars (D-fructose 98\%, D-glucose, D-sucrose 99.0\%, D-mannitol $98 \%$ and D-trehalose di-hydrate $99.5 \%$ ), tocopherols $(\alpha-, \quad \beta-, \quad \delta-, \quad$ and $\gamma$-isoforms), and trolox (6-hydroxy-2,5,7,8tetramethylchroman-2-carboxylic acid) were purchased from Sigma (St. Louis, MO, USA). Racemic tocol $50 \mathrm{mg} / \mathrm{ml}$, was purchased from Matreya (PA, USA). 2,2Diphenyl-1-picrylhydrazyl (DPPH) was obtained from Alfa Aesar (Ward Hill, MA, USA). All other chemicals and solvents were of analytical grade and purchased from common sources. Water was treated in a Milli-Q water purification system (TGI Pure Water Systems, USA).

\subsection{Evaluation of antioxidant activity}

\subsubsection{Preparation of the extracts}

Each sample ( $\sim 0.4 \mathrm{~g}$ for mycelia, roots and stems; $4 \mathrm{~g}$ for culture media) was extracted by stirring with $30 \mathrm{ml}$ of methanol $\left(25^{\circ} \mathrm{C}\right.$ at $\left.150 \mathrm{rpm}\right)$ for $1 \mathrm{~h}$ and subsequently filtered 
through Whatman No. 4 paper. The residue was then extracted under the same conditions. The combined methanolic extracts were evaporated at $40{ }^{\circ} \mathrm{C}$ (rotary evaporator Büchi R-210; Flawil, Switzerland) to dryness, and redissolved in methanol. In vitro assays already described by the authors (Barros et al., 2010) were used to evaluate the antioxidant activity of the samples.

\subsubsection{DPPH radical-scavenging activity}

This assay was performed in 96-well microtiter plates using an ELX800 Microplate Reader (Bio-Tek Instruments, Inc, Winooski, USA). The reaction mixture in each of the 96-wells of the plate consisted of one of the different concentrations of the extracts ( 30 $\mu \mathrm{l})$ and methanolic solution containing DPPH radicals $\left(6 \times 10^{-5} \mathrm{~mol} / \mathrm{l}, 270 \mu \mathrm{l}\right)$. The mixture was left to stand for $30 \mathrm{~min}$ in the dark. The reduction of the DPPH radical was determined by measuring the absorption at $515 \mathrm{~nm}$. The radical scavenging activity (RSA) was calculated as a percentage of DPPH discolouration using the equation: \% $\operatorname{RSA}=\left[\left(\mathrm{A}_{\mathrm{DPPH}}-\mathrm{A}_{\mathrm{S}}\right) / \mathrm{A}_{\mathrm{DPPH}}\right] \times 100$, where $\mathrm{A}_{\mathrm{S}}$ is the absorbance of the solution when the sample extract has been added at a particular level, and $\mathrm{A}_{\mathrm{DPPH}}$ is the absorbance of the DPPH solution. The extract concentration providing 50\% of radicals scavenging activity $\left(\mathrm{EC}_{50}\right)$ was calculated from the graph of RSA percentage against extract concentration. Trolox was used as standard.

\subsubsection{Reducing power}

This assay was also performed using microtiter plates and the Microplate Reader described above. Different concentrations of the extracts $(0.5 \mathrm{ml})$ were mixed with sodium phosphate buffer $(200 \mathrm{mmol} / \mathrm{l}, \mathrm{pH} 6.6,0.5 \mathrm{ml})$ and potassium ferricyanide $(1 \%$ $\mathrm{w} / \mathrm{v}, 0.5 \mathrm{ml}$ ). The mixture was incubated at $50{ }^{\circ} \mathrm{C}$ for $20 \mathrm{~min}$, and trichloroacetic acid 
$(10 \% \mathrm{w} / \mathrm{v}, 0.5 \mathrm{ml})$ was added. The mixture $(0.8 \mathrm{ml})$ was poured into the wells of a 48 well microplate, as also deionised water $(0.8 \mathrm{ml})$ and ferric chloride $(0.1 \% \mathrm{w} / \mathrm{v}, 0.16$ $\mathrm{ml}$ ), and the absorbance was measured at $690 \mathrm{~nm}$. The extract concentration providing 0.5 of absorbance $\left(\mathrm{EC}_{50}\right)$ was calculated from the graph of absorbance at $690 \mathrm{~nm}$ against extract concentration. Trolox was used as standard.

\subsubsection{Inhibition of $\beta$-carotene bleaching}

A solution of $\beta$-carotene was prepared by dissolving $\beta$-carotene $(2 \mathrm{mg})$ in chloroform $(10 \mathrm{ml})$. Two millilitres of this solution were pipetted into a round-bottom flask. The chloroform was removed at $40{ }^{\circ} \mathrm{C}$ under vacuum and linoleic acid (40 mg), Tween 80 emulsifier (400 mg), and distilled water $(100 \mathrm{ml})$ were added to the flask with vigorous shaking. Aliquots $(4.8 \mathrm{ml})$ of this emulsion were transferred into test tubes containing different concentrations of the extracts $(0.2 \mathrm{ml})$. The tubes were shaken and incubated at $50{ }^{\circ} \mathrm{C}$ in a water bath. As soon as the emulsion was added to each tube, the zero time absorbance was measured at $470 \mathrm{~nm}$ (Analytikjena 200 spectrophotometer). $\beta$-Carotene bleaching inhibition was calculated using the following equation: $(\beta$-carotene content after $2 \mathrm{~h}$ of assay/initial $\beta$-carotene content $) \times 100$. The extract concentration providing $50 \%$ antioxidant activity $\left(\mathrm{EC}_{50}\right)$ was calculated by interpolation from the graph of $\beta$ carotene bleaching inhibition percentage against extract concentration. Trolox was used as standard.

2.4.5. Inhibition of lipid peroxidation using thiobarbituric acid reactive substances (TBARS) 
Brains were obtained from porcine (Sus scrofa), dissected, and homogenized with a Polytron in ice cold Tris- $\mathrm{HCl}$ buffer $(20 \mathrm{mM}$, $\mathrm{pH} 7.4)$ to produce a 1:2 w/v brain tissue homogenate which was centrifuged at $3000 \mathrm{~g}$ for10 $\mathrm{min}$. An aliquot $(0.1 \mathrm{ml})$ of the supernatant was incubated with the different concentrations of the samples solutions $(0.2 \mathrm{ml})$ in the presence of $\mathrm{FeSO}_{4}(10 \mathrm{mM} ; 0.1 \mathrm{ml})$ and ascorbic acid $(0.1 \mathrm{mM} ; 0.1 \mathrm{ml})$ at $37^{\circ} \mathrm{C}$ for $1 \mathrm{~h}$. The reaction was stopped by the addition of trichloroacetic acid $(28 \%$ $\mathrm{w} / \mathrm{v}, 0.5 \mathrm{ml}$ ), followed by thiobarbituric acid (TBA, $2 \%$, w/v, $0.38 \mathrm{ml}$ ), and the mixture was then heated at $80^{\circ} \mathrm{C}$ for $20 \mathrm{~min}$. After centrifugation at $3000 \mathrm{~g}$ for $10 \mathrm{~min}$ to remove the precipitated protein, the colour intensity of the malondialdehyde (MDA)-TBA complex in the supernatant was measured by its absorbance at $532 \mathrm{~nm}$. The inhibition ratio $(\%)$ was calculated using the following formula: Inhibition ratio $(\%)=[(\mathrm{A}-\mathrm{B}) / \mathrm{A}]$ $\times 100 \%$, where $\mathrm{A}$ and $\mathrm{B}$ were the absorbance of the control and the sample solution, respectively. The extract concentration providing 50\% lipid peroxidation inhibition $\left(\mathrm{EC}_{50}\right)$ was calculated by interpolation from the graph of TBARS formation inhibition percentage against extract concentration. Trolox was used as standard.

\subsection{Determination of antioxidant compounds}

\subsubsection{Total phenolics}

An aliquot of the extract solution $(0.5 \mathrm{ml})$ was mixed with Folin-Ciocalteu (FC) reagent (2.5 $\mathrm{ml}$, previously diluted with water $1: 10 \mathrm{v} / \mathrm{v})$ and sodium carbonate $(75 \mathrm{~g} / 1,2 \mathrm{ml})$. The tubes were vortexed for $15 \mathrm{~s}$ and allowed to stand for $30 \mathrm{~min}$ at $40{ }^{\circ} \mathrm{C}$ for colour development. Absorbance was then measured at $765 \mathrm{~nm}$. Gallic acid was used to calculate the standard curve $(0.05-0.8 \mathrm{mM})$, and the results were expressed as $\mathrm{mg}$ of gallic acid equivalents (GAEs) per g of extract. 


\subsubsection{Tocopherols}

Tocopherols content was determined following a procedure previously optimized and described by Barros et al. (2008), using tocol as internal standard. The HPLC equipment consisted of an integrated system with a Smartline 1000 pump (Knauer, Berlin, Germany), a Smartline manager 5000 degasser, an AS-2057 auto-sampler (Jasco, Easton, MD) and a FP-2020 fluorescence detector (Jasco, Easton, MD) programmed for excitation at $290 \mathrm{~nm}$ and emission at $330 \mathrm{~nm}$.. Data were analysed using Clarity 2.4 Software (DataApex). The column used was a normal-phase $250 \mathrm{~mm} \times 4.6 \mathrm{~mm}$ i.d., 5 $\mu \mathrm{m}$, Polyamide II, with a $10 \mathrm{~mm} \times 4 \mathrm{~mm}$ i.d. guard column of the same material (YMC Waters, Dinslaken, Germany), operating at $30^{\circ} \mathrm{C}$. The mobile phase used was a mixture of $n$-hexane and ethyl acetate $(70: 30, \mathrm{v} / \mathrm{v})$ at a flow rate of $1 \mathrm{ml} / \mathrm{min}$. Tocopherols identification was made by comparing the relative retention times of sample peaks with standards. Quantification was based on the fluorescence signal response, using the internal standard method, and the results were expressed in mg per $g$ of dry weight $(\mathrm{dw})$.

\subsubsection{Free sugars}

Sugars content was determined following a procedure previously optimized and described by Heleno et al. (2009), using raffinose or melizitose as internal standards for mycelium and plant analyses, respectively. The HPLC system described above was connected to a Smartline 2300 refraction index (RI) detector (Knauer). Data were analysed using Clarity 2.4 Software (DataApex). The chromatographic separation was achieved with a Eurospher 100-5 $\mathrm{NH}_{2}$ column $(4.6 \times 250 \mathrm{~mm}, 5 \mathrm{~mm}$, Knauer $)$ operating at $30^{\circ} \mathrm{C}$. The mobile phase was acetonitrile/deionized water, 70:30 (v/v) at a flow rate of $1 \mathrm{ml} / \mathrm{min}$. Sugars identification was made by comparing the relative retention times of 
sample peaks with standards. Quantification was made by the internal standard method and the results were expressed in g per $100 \mathrm{~g}$ of dry weight (dw).

\subsection{Statistical analysis}

An analysis of variance (ANOVA) with Type III sums of squares was performed using the GLM (General Linear Model) procedure of the SPSS software, version 18.0 (SPSS, Inc.). All dependent variables were analyzed using a 2-way ANOVA, being the main factors the "culture element" (C. sativa root, C. sativa stem, mycelium and culture medium) and the "co-culture period" $(0,6,24$ and $72 \mathrm{~h})$. Since a statistical significant interaction effect ("culture element×co-culture period") was found in all tests, the two factors were evaluated simultaneously by plotting the estimated marginal means for all levels of each factor. In addition, a linear discriminant analysis (LDA) was used as a technique to classify the four culture elements (CE) as well as the four co-culture periods (CP) according to their antioxidant activity results, and their phenolics, sugars and tocopherols contents. A stepwise technique, using the Wilks' $\lambda$ method with the usual probabilities of $F$ (3.84 to enter and 2.71 to remove), was applied for variable selection. This procedure uses a combination of forward selection and backward elimination procedures, where before selecting a new variable to be included, it is verified whether all variables previously selected remain significant (Rencher, 1995; Maroco, 2003; López et al., 2008). With this approach, it is possible to identify the significant variables among the antioxidant activity, and phenolics, sugars and tocopherols profiles obtained for each sample. To verify which canonical discriminant functions were significant, the Wilks' $\lambda$ test was applied. To avoid overoptimistic data modulation, a leaving-one-out cross-validation procedure was carried out to assess the 
model performance. The LDA statistical analysis and the other statistical tests were performed at a 5\% significance level using the SPSS software mentioned above.

\section{Results and Discussion}

Antioxidant activity $\mathrm{EC}_{50}$ values and phenolics (Table 1), tocopherols (Table 2) and sugars (Table 3) contents are reported as mean value of each co-culture element (CE) over the different co-culture periods (CP), as well as mean value of all elements within each co-culture period. Despite some eventual loss of information, when the results are presented in this way, the effects of both factors (CE and $\mathrm{CP}$ ) are more accurately evaluated, since their interaction influence is avoided. In fact, the results showed that the interaction $\mathrm{CP} \times \mathrm{CE}$ was a significant $(p<0.001)$ source of variation for all the evaluated parameters. Hence, the multiple comparisons results, usually obtained after Tukey HSD post hoc test, could not be presented. Nevertheless, from the analysis of the least square means together with the plots (data not shown) of the estimated marginal means (EMM) it was possible to identify specific tendencies according with particular variables.

In $P$. arhizus-C. sativa co-culture, the culture medium was the element with the lowest antioxidant activity (highest $\mathrm{EC}_{50}$ values) for all the performed assays. Accordingly, this was also the element with the lowest values of phenolic content (4 mg GAE/g extract). Otherwise, $C$. sativa roots and stems revealed the highest antioxidant activity $(\leq 0.05$ $\mathrm{mg} / \mathrm{ml}$ ) and phenolic contents ( $\geq 537 \mathrm{mg}$ GAE/g extract). Although this conclusion could not be drawn directly from Table 1, after EMM plots analysis it is clear that the antioxidant activity of $C$. sativa roots and stems and $P$. arhizus mycelium remained nearly constant from 0 to $72 \mathrm{~h}$, being the observed differences mainly related with the culture media (DPPH scavenging activity increased and TBARS inhibition decreased), 
showing a similar behavior to that obtained when the fungus was co-cultivated with $P$. pinaster (Carocho et al., 2012).

In $P$. involutus-C. sativa co-culture, the results indicate a completely different association. In fact, the $\mathrm{EC}_{50}$ values obtained for $\mathrm{DPPH}$ scavenging activity, reducing power and $\beta$-carotene bleaching assays were higher in $C$. sativa stems and roots. This result might indicate a greater compatibility for this mycorrhizal association, unless in the singular case of TBARS assay. The $P$. involutus mycelium was the most antioxidant $\mathrm{CE}$ in all assays. Nevertheless, its antioxidant activity seemed to be related not only with phenolics content, since this $\mathrm{CE}$ did not reveal the highest amount of these compounds. The $\mathrm{EC}_{50}$ values obtained for the culture medium were lower than those obtained for the same element for the $P$. arhizus-C. sativa co-culture, in agreement with the reported by Reis et al. $(2011,2012)$ and Carocho et al. (2012) and with its higher phenolic content (Table 1). Considering co-culture period (CP), this mycorrhization showed higher compatibility after $24 \mathrm{~h}$, as the highest $\mathrm{EC}_{50}$ values at this time indicate, especially in the cases of DPPH radical scavenging activity, $\beta$-carotene bleaching assay or TBARS formation inhibition. However, the phenolics content was also highest in this period, indicating that the antioxidant activity is not only correlated to phenolics, but also with other antioxidant compounds. Along time, $P$. involutus mycelium and culture medium were the $\mathrm{CE}$ with the highest antioxidant activity variation. These results clearly indicate compatibility differences among the two fungi. P. involutus seemed to be more compatible with $C$. sativa, revealing that the chemical interactions have a high dependency on the used plant. It should be reminded that when the co-culture was assayed with $P$. pinaster precisely for the same periods $(0,6,24$ and $72 \mathrm{~h})$, the most compatible fungus was $P$. arhizus Carocho et al. (2012). 
Considering tocopherols contents in $P$. arhizus-C. sativa co-culture, the mycelium clearly dominates tocopherols concentration (Table 2) due to its high $\gamma$-tocopherol values $(47418 \pm 5934 \mu \mathrm{g} / 100 \mathrm{~g} \mathrm{dw})$. Along time, some particular tendencies could be found: $\alpha$-tocopherol decreased in $C$. sativa stems and roots; $\beta$-tocopherol and $\delta$ tocopherol decreased in C. sativa roots (Figure 2A).

Regarding P. involutus-C. sativa co-culture, the plots of the EMM indicated that $\alpha-, \beta-$ tocopherol and total tocopherols decreased in C. sativa stems and roots. Comparing with P. arhizus-C. sativa co-culture, $\gamma$-tocopherol revealed much lower contents, especially due to its concentration in $P$. involutus mycelium, which is quite lower than in $P$. arhizus. Nevertheless, $\gamma$-tocopherol increase slightly in P. involutus mycelium, while $\delta$ tocopherol showed a marked decrease in C. sativa roots. Considering total tocopherols, the most obvious results are their decrease in C. sativa parts.

$\alpha$-Tocopherol was higher in $C$. sativa stems and $\beta$-tocopherol was prevalent in $C$. sativa roots in both co-cultures. Mycorrhization with both ECM fungi seemed not to stimulate the production of tocopherols in roots and stems.

Regarding sugars contents, the elements of both co-cultures presented similar profiles, except for mannitol, which was only detected in $P$. involutus mycelium (Figure 2B). Curiously, this sugar alcohol derivative was found only in $P$. arhizus mycelium when the fungus was mycorrhized with $P$. pinaster (Carocho et al., 2012). This finding strongly indicates that, once more, chemical interactions are dependent of the co-culture elements. It should be highlighted that mannitol was present in the most compatible ECM of each study; this finding needs further experiments in order to confirm the crucial role of this primary metabolite in the compatibility dynamics. Fructose and sucrose were only found in C. sativa roots and stems, in both co-cultures, and in similar 
contents (Table 2B). The tendencies verified for sucrose (increased along time in $C$. sativa stems), glucose (decreased along time in culture media), trehalose (decreased along time in mycelia) and total sugars (decreased along time in culture media) were identical in both co-cultures.

Since some particular differences cannot be directly observed in tables, a linear discriminant analysis (LDA) was applied to better comprehend the differences brought on by co-culture period or elements. The differences induced by the co-culture period (CP) for the mycorrhization P. arhizus-C. sativa allowed the individualization of four clusters (corresponding to the four assayed periods) (Figure 3A). To achieve this separation all the assayed parameters (except total tocopherols, total sugars and, obviously, mannitol) were included in the LDA, since the attempts with less variables did not show discriminant ability. The obtained discriminant model include three significant $(p<0.001$ for the Wilks' $\lambda$ test $)$ discriminant functions. These three functions explained $100.0 \%$ of the variance of the experimental data (the first explained 95.1\%, the second 3.0\% and the third 1.9\%) (Figure 3A). The model showed a very satisfactory classification performance allowing to correctly classifying $98.6 \%$ of the samples for the original groups and $96.5 \%$ for the cross-validation procedure.

The discrimination efficiency was higher when applied to separate co-culture elements. The applied LDA also allowed the formation of four individual clusters, but merely based in sugars contents. Three significant discriminant functions were also defined, explaining $100.0 \%$ of the variance of the experimental data (the first explained $85.3 \%$, the second $13.9 \%$ and the third $0.8 \%$ ) (Figure 3B). The model classified correctly $100.0 \%$ of the samples for the original groups and for the cross-validation procedure.

Regarding $P$. involutus-C. sativa co-culture, the results obtained for the assayed CP seemed to contain higher differences than the formerly described co-culture. When the 
LDA was performed using all the assayed parameters (except reducing power, total sugars and total tocopherols), the obtained model classified properly $100.0 \%$ of the samples for the original groups and $99.3 \%$ for the cross-validation procedure. This model was based in three significant discriminant functions that comprised $100.0 \%$ of the observed variance (the first explained $82.74 \%$, the second $15.22 \%$ and the third 2.04\%). The different profiles obtained for each CE, similarly to the P. arhizus-C. sativa co-culture, proved to be more accurate in defining the individual clusters correspondent to the naturally occurring groups (Figure 4A). This last model defined also three significant discriminant functions that include $100.0 \%$ of the observed variance (the first explained $85.3 \%$, the second $13.9 \%$ and the third $0.8 \%$ ). The classification performance was very satisfactory for all the assayed combinations of parameters, but due to the easiness of appliance in future works, the exhibited plot (Figure 4B) refers to the discriminant scores obtained according with the results of sugars profiles.

Overall, sugars profiles were the most discriminant parameters, either considering $\mathrm{CP}$ or CE, for both co-cultures. The differences observed along time might indicate that the chemical interactions are mainly related with this class of compounds, particularly with mannitol. $P$. involutus seemed to be the most adequate fungus to mycorrhize with $C$. sativa. On the other hand, and considering bioactive compounds production, P. arhizus was more efficient since allowed an increase in the contents of sugars and tocopherols in all co-culture elements.

\section{Acknowledgements}


The authors are grateful to the Foundation for Science and Technology (FCT, Portugal) and COMPETE/QREN/EU for financial support through the research project PTDC/AGR-ALI/110062/2009. L. Barros and J.C.M. Barreira thank to FCT, POPHQREN and FSE for their grants (SFRH/BPD/4609/2008 and SFRH/BPD/72802/2010, respectively).

\section{References}

Agricultural Statistics. Instituto Nacional de Estatística I. P., 2010, Lisboa, Portugal.

Baptista, P., Martins, A., Pais, M.S., Tavares, R.M., Lino-Neto, T. 2007. Involvement of reactive oxygen species during early stages of ectomycorrhiza establishment between Castanea sativa and Pisolithus tinctorius. Mycorrhiza 17, 185-193.

Baptista, P., Martins, A., Tavares, R.M., Lino-Neto, T. 2010. Diversity and fruiting pattern of macrofungi associated with chestnut (Castanea sativa) in the Trás-osMontes region (Northeast Portugal). Fungal Ecology, 3, 9-19.

Barreira J. C. M.; Ferreira I. C. F. R.; Oliveira M. B. P. P.; Pereira J. A. Antioxidant activity of the extracts from chestnut flower, leaf, skins and fruit. Food Chemistry, 2008, 107, 1106-1113.

Barros, L., Correia, D.M., Ferreira, I.C.F.R., Baptista, P., Santos-Buelga, C. 2008. Optimization of the determination of tocopherols in Agaricus sp. edible mushrooms by a normal phase liquid chromatographic method. Food Chem. 4, 1046-1050.

Barros, L., Oliveira, S., Carvalho, A.M., Ferreira, I.C.F.R. 2010. In vitro antioxidant properties and characterization in nutrients and phytochemicals of six medicinal plants from the Portuguese folk medicine. Ind. Crops Prod. 32, 572-579.

Branzanti MB, Rocca E, Pisi A, 1999. Effect of ectomycorrhizal fungi on chestnut ink disease. Mycorrhiza 9: 103-109. 
Carocho, M., Ferreira, I.C.F.R., Barros, L., Barreira, J.C.M., Martins, A. 2012. Antioxidants in Pinus Pinaster roots and mycorrhizal fungi during the early steps of symbiosis. Ind. Crops Prod. 38, 99-106.

Harvengt, L. 2005. Somatic embryogenesis in maritime pine (Pinus pinaster Ait). Foresty Sci. 77, 107-119.

Heleno, S.A., Barros, L., Sousa, M.J., Martins, A., Ferreira, I.C.F.R. 2009. Study and characterization of selected nutrients in wild mushrooms from Portugal by gas chromatography and high performance liquid chromatography. Microchem. J. 93, 195-199.

Lamb, C, Dixon, R.A. 1997. The oxidative burst in plant disease resistance. Annu. Rev. Plant Physiol. Plant Mol. Biol. 48, 251-275.

López, A., García, P., Garrido, A. 2008. Multivariate characterization of table olives according to their mineral nutrient composition, Food Chem. 106, 369-378.

Maroco, J. 2003. Análise Estatística, com utilização do SPSS, Edições Sílabo, Lisboa, Portugal.

Martins A, 1997. Micorrização in vitro de plantas micropropagadas de castanheiro (Castanea sativa Mill). Seérie de Estudos Escola Superior Agrária. Instituto Politécnico de Bragança, Bragança, Portugal.

Martins A, Casimiro A, Pais MMS,1997. Influence of mycorrhization on physiological parameters of micropropagated Castanea sativa Mill. plants. Mycorrhiza 7: 161-165.

Marx, D.H. 1969. The influence of ectotrophic fungi on the resistance of pine root to pathogenic infections. I. Antagonism of mycorrhizal fungi to root pathogenic fungi and soil bacteria. Phytopathol. 59, 153-163.

Münzenberger, B., Kottke, I., Oberwinkler, F. 1995. Reduction of phenolics in mycorrhizas of Larix decidua Mill. Tree Physiol. 15, 191-196. 
Münzenberger, B., Otter, T., Wüstrich, D., Polle, A. 1997. Peroxidase and laccase activities in mycorrhizal and non-mycorrhizal fine roots of Norway spruce (Picea abies) and larch (Larix decidua). Can. J. Bot. 78, 932-938.

Reis, F.S., Ferreira, I.C.F.R., Barros, L., Santos-Buelga, C., Martins, A. 2011. Mycorrhizal induction of phenolic compounds and antioxidant properties of fungi and seedlings during the early steps of symbiosis. Chemoecology 21, 151-159.

Reis, F.S., Ferreira, I.C.F.R., Martins, A. 2012. Effect of the mycorrhizal symbiosis time in the antioxidant activity of fungi and Pinus pinaster roots, stems and leaves. Ind. Crops Prod. 35, 211-216.

Rencher, A.C. 1995. Methods of Multivariate Analysis, John Willey, New York. 
Table 1. Antioxidant activity $\mathrm{EC}_{50}$ values $(\mathrm{mg} / \mathrm{ml})$ and phenolic content $(\mathrm{mg} \mathrm{GAE} / \mathrm{g}$ extract) obtained for the culture elements $(\mathrm{CE})$. The results are presented as mean $\pm \mathrm{SD}^{\mathrm{a}}$ $(n=36$, for each co-culture period (CP) and for each CE).

\begin{tabular}{|c|c|c|c|c|c|c|}
\hline & & $\begin{array}{c}\text { DPPH } \\
\text { scavenging } \\
\text { activity }\end{array}$ & $\begin{array}{l}\text { Reducing } \\
\text { power }\end{array}$ & $\begin{array}{l}\beta \text {-Carotene } \\
\text { bleaching } \\
\text { inhibition }\end{array}$ & $\begin{array}{l}\text { TBARS } \\
\text { formation } \\
\text { inhibition }\end{array}$ & Phenolics \\
\hline \multicolumn{7}{|c|}{ Pisolithus arhizus } \\
\hline \multirow{5}{*}{$\mathrm{CP}$} & $0 \mathrm{~h}$ & $20 \pm 34$ & $6 \pm 10$ & $1 \pm 1$ & $0.1 \pm 0.2$ & $378 \pm 312$ \\
\hline & $6 \mathrm{~h}$ & $20 \pm 34$ & $5 \pm 9$ & $5 \pm 8$ & $1 \pm 2$ & $189 \pm 150$ \\
\hline & $24 \mathrm{~h}$ & $26 \pm 43$ & $9 \pm 16$ & $17 \pm 29$ & $1 \pm 2$ & $483 \pm 444$ \\
\hline & $72 \mathrm{~h}$ & $11 \pm 18$ & $5 \pm 9$ & $1 \pm 2$ & $1 \pm 2$ & $285 \pm 252$ \\
\hline & $p$-value & $<0.001$ & $<0.001$ & $<0.001$ & $<0.001$ & $<0.001$ \\
\hline \multirow{5}{*}{$\mathrm{CE}$} & Root & $0.05 \pm 0.05$ & $0.04 \pm 0.03$ & $0.03 \pm 0.02$ & $0.01 \pm 0.02$ & $671 \pm 263$ \\
\hline & Stem & $0.04 \pm 0.03$ & $0.04 \pm 0.03$ & $0.1 \pm 0.1$ & $0.01 \pm 0.02$ & $537 \pm 203$ \\
\hline & Medium & $74 \pm 22$ & $25 \pm 6$ & $23 \pm 26$ & $3 \pm 2$ & $4 \pm 2$ \\
\hline & Mycelium & $2 \pm 1$ & $0.5 \pm 0.2$ & $0.5 \pm 0.2$ & $0.06 \pm 0.03$ & $122 \pm 40$ \\
\hline & $p$-value & $<0.001$ & $<0.001$ & $<0.001$ & $<0.001$ & $<0.001$ \\
\hline $\mathrm{CP} \times \mathrm{CE}$ & $p$-value & $<0.001$ & $<0.001$ & $<0.001$ & $<0.001$ & $<0.001$ \\
\hline \multicolumn{7}{|c|}{ Paxillus involutus } \\
\hline \multirow{5}{*}{$\mathrm{CP}$} & $0 \mathrm{~h}$ & $4 \pm 3$ & $4 \pm 4$ & $9 \pm 10$ & $3 \pm 4$ & $377 \pm 312$ \\
\hline & $6 \mathrm{~h}$ & $25 \pm 23$ & $19 \pm 20$ & $10 \pm 8$ & $1 \pm 2$ & $481 \pm 375$ \\
\hline & $24 \mathrm{~h}$ & $43 \pm 38$ & $45 \pm 45$ & $101 \pm 98$ & $0.07 \pm 0.04$ & $245 \pm 190$ \\
\hline & $72 \mathrm{~h}$ & $15 \pm 14$ & $19 \pm 19$ & $14 \pm 11$ & $0.4 \pm 0.6$ & $513 \pm 391$ \\
\hline & $p$-value & $<0.001$ & $<0.001$ & $<0.001$ & $<0.001$ & $<0.001$ \\
\hline \multirow{5}{*}{$\mathrm{CE}$} & Root & $34 \pm 26$ & $31 \pm 21$ & $66 \pm 84$ & $0.05 \pm 0.02$ & $783 \pm 212$ \\
\hline & Stem & $45 \pm 30$ & $51 \pm 39$ & $59 \pm 74$ & $0.5 \pm 0.6$ & $621 \pm 164$ \\
\hline & Medium & $8 \pm 3$ & $5 \pm 4$ & $9 \pm 3$ & $4 \pm 4$ & $12 \pm 6$ \\
\hline & Mycelium & $0.3 \pm 0.2$ & $0.2 \pm 0.1$ & $0.5 \pm 0.2$ & $0.01 \pm 0.01$ & $200 \pm 58$ \\
\hline & $p$-value & $<0.001$ & $<0.001$ & $<0.001$ & $<0.001$ & $<0.001$ \\
\hline $\mathrm{CP} \times \mathrm{CE}$ & $p$-value & $<0.001$ & $<0.001$ & $<0.001$ & $<0.001$ & $<0.001$ \\
\hline
\end{tabular}


${ }^{a}$ Results are reported as mean value of each culture element (CE) over the different coculture periods (CP) as well as mean value of all elements within each co-culture period. Therefore, SD reflects values in those samples (with different CE or CP), and can be higher than mean values. 
Table 2. Tocopherols content $(\mu \mathrm{g} / 100 \mathrm{~g} \mathrm{dw})$ in the culture elements (CE). The results are presented as mean $\pm \mathrm{SD}^{\mathrm{a}}(\mathrm{n}=36$, for each co-culture period (CP) and for each $\mathrm{CE})$.

$\alpha$-Tocopherol $\beta$-Tocopherol $\quad \gamma$-Tocopherol $\delta$-Tocopherol $\quad$ Total tocopherols

\begin{tabular}{|c|c|c|c|c|c|c|}
\hline \multicolumn{7}{|c|}{ Pisolithus arhizus } \\
\hline \multirow{5}{*}{$\mathrm{CP}$} & $0 \mathrm{~h}$ & $1989 \pm 2115$ & $195 \pm 143$ & $11025 \pm 18573$ & $94 \pm 87$ & $13297 \pm 17592$ \\
\hline & $6 \mathrm{~h}$ & $347 \pm 348$ & $88 \pm 80$ & $14342 \pm 24773$ & $58 \pm 13$ & $14834 \pm 24618$ \\
\hline & $24 \mathrm{~h}$ & $361 \pm 366$ & $120 \pm 88$ & $10861 \pm 18516$ & $43 \pm 8$ & $11385 \pm 18400$ \\
\hline & $72 \mathrm{~h}$ & $417 \pm 534$ & $101 \pm 67$ & $12159 \pm 20904$ & $47 \pm 30$ & $12724 \pm 20753$ \\
\hline & $p$-value & $<0.001$ & $<0.001$ & $<0.001$ & $<0.001$ & 0.001 \\
\hline \multirow{5}{*}{$\mathrm{CE}$} & Roots & $1073 \pm 1147$ & $224 \pm 84$ & $170 \pm 80$ & $74 \pm 83$ & $1538 \pm 1378$ \\
\hline & Stems & $1990 \pm 1716$ & $59 \pm 41$ & $659 \pm 252$ & $86 \pm 32$ & $2790 \pm 2020$ \\
\hline & Medium & $2 \pm 1$ & $20 \pm 15$ & $140 \pm 82$ & $37 \pm 24$ & $199 \pm 117$ \\
\hline & Mycelium & $50 \pm 17$ & $201 \pm 73$ & $47418 \pm 5934$ & $45 \pm 14$ & $47714 \pm 5890$ \\
\hline & $p$-value & $<0.001$ & $<0.001$ & $<0.001$ & $<0.001$ & $<0.001$ \\
\hline $\mathrm{CP} \times \mathrm{CE}$ & $p$-value & $<0.001$ & $<0.001$ & $<0.001$ & $<0.001$ & $<0.001$ \\
\hline \multicolumn{7}{|c|}{ Paxillus involutus } \\
\hline \multirow{5}{*}{$\mathrm{CP}$} & $0 \mathrm{~h}$ & $1985 \pm 2120$ & $119 \pm 146$ & $479 \pm 400$ & $103 \pm 81$ & $2683 \pm 2559$ \\
\hline & $6 \mathrm{~h}$ & $473 \pm 495$ & $27 \pm 31$ & $649 \pm 881$ & $49 \pm 33$ & $1199 \pm 829$ \\
\hline & $24 \mathrm{~h}$ & $541 \pm 676$ & $39 \pm 39$ & $490 \pm 523$ & $94 \pm 76$ & $1165 \pm 876$ \\
\hline & $72 \mathrm{~h}$ & $678 \pm 766$ & $89 \pm 106$ & $585 \pm 713$ & $27 \pm 21$ & $1378 \pm 913$ \\
\hline & $p$-value & $<0.001$ & $<0.001$ & $<0.001$ & $<0.001$ & 0.001 \\
\hline \multirow{5}{*}{$\mathrm{CE}$} & Roots & $1215 \pm 1067$ & $191 \pm 119$ & $121 \pm 100$ & $103 \pm 67$ & $1629 \pm 1327$ \\
\hline & Stems & $2413 \pm 1480$ & $82 \pm 35$ & $591 \pm 291$ & $86 \pm 41$ & $3173 \pm 1822$ \\
\hline & Medium & $12 \pm 8$ & nd & $43 \pm 25$ & nd & $55 \pm 32$ \\
\hline & Mycelium & $37 \pm 21$ & nd & $1448 \pm 600$ & $83 \pm 72$ & $1568 \pm 607$ \\
\hline & $p$-value & $<0.001$ & $<0.001$ & $<0.001$ & $<0.001$ & $<0.001$ \\
\hline $\mathrm{CP} \times \mathrm{CE}$ & $p$-value & $<0.001$ & $<0.001$ & $<0.001$ & $<0.001$ & $<0.001$ \\
\hline
\end{tabular}


${ }^{a}$ Results are reported as mean value of each culture element (CE) over the different coculture periods $(\mathrm{CP})$ as well as mean value of all elements within each co-culture period. Therefore, SD reflects values in those samples (with different CE or CP), and can be higher than mean values. 
Table 3. Sugars content $(\mathrm{g} / 100 \mathrm{~g} \mathrm{dw})$ in the culture elements $(\mathrm{CE})$. The results are presented as mean $\pm \mathrm{SD}^{\mathrm{a}}$ ( $\mathrm{n}=36$, for each co-culture period $(\mathrm{CP})$ and for each $\mathrm{CE}$ ).

\begin{tabular}{|c|c|c|c|c|c|c|c|}
\hline & & Fructose & Glucose & Mannitol & Sucrose & Trehalose & Total sugars \\
\hline & & \multicolumn{6}{|c|}{ Pisolithus arhizus } \\
\hline \multirow{5}{*}{$\mathrm{CP}$} & $0 \mathrm{~h}$ & $0.2 \pm 0.3$ & $6 \pm 9$ & nd & $0.4 \pm 0.6$ & $1 \pm 2$ & $8 \pm 8$ \\
\hline & $6 \mathrm{~h}$ & $0.1 \pm 0.1$ & $5 \pm 7$ & nd & $1 \pm 1$ & $1 \pm 1$ & $6 \pm 6$ \\
\hline & $24 \mathrm{~h}$ & $0.1 \pm 0.2$ & $5 \pm 7$ & nd & $1 \pm 1$ & $0.4 \pm 0.4$ & $6 \pm 6$ \\
\hline & $72 \mathrm{~h}$ & $0.3 \pm 0.4$ & $4 \pm 6$ & nd & $1 \pm 1$ & $0.3 \pm 0.4$ & $6 \pm 6$ \\
\hline & $p$-value & $<0.001$ & $<0.001$ & - & $<0.001$ & $<0.001$ & $<0.001$ \\
\hline \multirow{5}{*}{$\mathrm{CE}$} & Roots & $0.2 \pm 0.1$ & $0.2 \pm 0.1$ & nd & $0.6 \pm 0.4$ & $0.2 \pm 0.1$ & $1.2 \pm 0.4$ \\
\hline & Stems & $0.6 \pm 0.2$ & $0.5 \pm 0.2$ & nd & $3 \pm 1$ & $0.17 \pm 0.01$ & $4 \pm 1$ \\
\hline & Medium & nd & $18 \pm 2$ & nd & nd & nd & $18 \pm 2$ \\
\hline & Mycelium & nd & $1 \pm 1$ & nd & nd & $2 \pm 2$ & $3 \pm 2$ \\
\hline & $p$-value & $<0.001$ & $<0.001$ & - & $<0.001$ & $<0.001$ & $<0.001$ \\
\hline \multirow[t]{2}{*}{$\mathrm{CP} \times \mathrm{CE}$} & $p$-value & $<0.011$ & $<0.001$ & - & $<0.001$ & $<0.001$ & $<0.001$ \\
\hline & & & \multicolumn{5}{|c|}{ Paxillus involutus } \\
\hline \multirow{5}{*}{$\mathrm{CP}$} & $0 \mathrm{~h}$ & $0.2 \pm 0.3$ & $7 \pm 11$ & $0.2 \pm 0.3$ & $0.4 \pm 0.6$ & $0.2 \pm 0.2$ & $8 \pm 11$ \\
\hline & $6 \mathrm{~h}$ & $0.2 \pm 0.2$ & $6 \pm 8$ & $2 \pm 3$ & $1 \pm 1$ & $0.2 \pm 0.2$ & $8 \pm 7$ \\
\hline & $24 \mathrm{~h}$ & $0.1 \pm 0.1$ & $6 \pm 9$ & $2 \pm 3$ & $1 \pm 1$ & $0.2 \pm 0.2$ & $9 \pm 8$ \\
\hline & $72 \mathrm{~h}$ & $0.2 \pm 0.2$ & $5 \pm 7$ & $1 \pm 2$ & $1 \pm 1$ & $0.1 \pm 0.1$ & $7 \pm 6$ \\
\hline & $p$-value & $<0.001$ & $<0.001$ & $<0.001$ & $<0.001$ & $<0.001$ & $<0.001$ \\
\hline \multirow{5}{*}{$\mathrm{CE}$} & Roots & $0.3 \pm 0.1$ & $0.4 \pm 0.1$ & nd & $0.5 \pm 0.4$ & $0.10 \pm 0.02$ & $1.3 \pm 0.4$ \\
\hline & Stems & $0.5 \pm 0.1$ & $0.5 \pm 0.1$ & nd & $2.1 \pm 0.4$ & $0.19 \pm 0.02$ & $3.3 \pm 0.3$ \\
\hline & Medium & nd & $21 \pm 3$ & nd & nd & nd & $21 \pm 3$ \\
\hline & Mycelium & nd & $0.8 \pm 0.3$ & $5 \pm 3$ & nd & $0.5 \pm 0.1$ & $6 \pm 2$ \\
\hline & $p$-value & $<0.001$ & $<0.001$ & $<0.001$ & $<0.001$ & $<0.001$ & $<0.001$ \\
\hline $\mathrm{CP} \times \mathrm{CE}$ & $p$-value & $<0.011$ & $<0.001$ & $<0.001$ & $<0.001$ & $<0.001$ & $<0.001$ \\
\hline
\end{tabular}

\footnotetext{
${ }^{a}$ Results are reported as mean value of each culture element (CE) over the different coculture periods (CP) as well as mean value of all elements within each co-culture
} 
period. Therefore, SD reflects values in those samples (with different $\mathrm{CE}$ or $\mathrm{CP}$ ), and can be higher than mean values. 

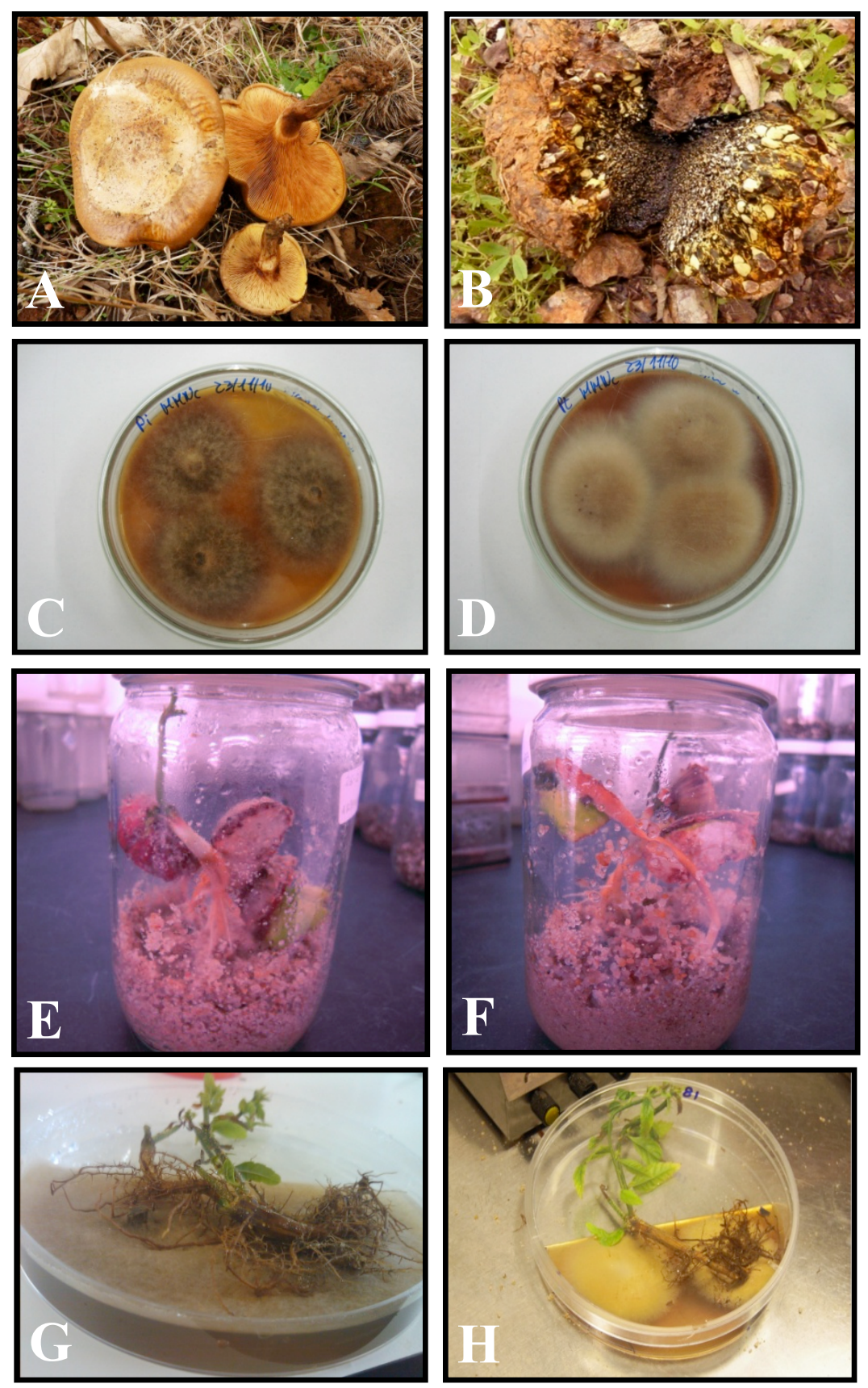

Figure 1. A. Paxillus involutus fruiting body; B. Pisolithus arhizus fruiting body; C. Paxillus involutus mycelium; D. Pisolithus arhizus mycelium; E. and F. Castanea sativa seedlings in different stages; G - Paxillus involutus mycelium- Castanea sativa seedlings mycorrhizal induction in vitro; H. Pisolithus arhizus mycelium- Castanea sativa seedlings mycorrhizal induction in vitro. 


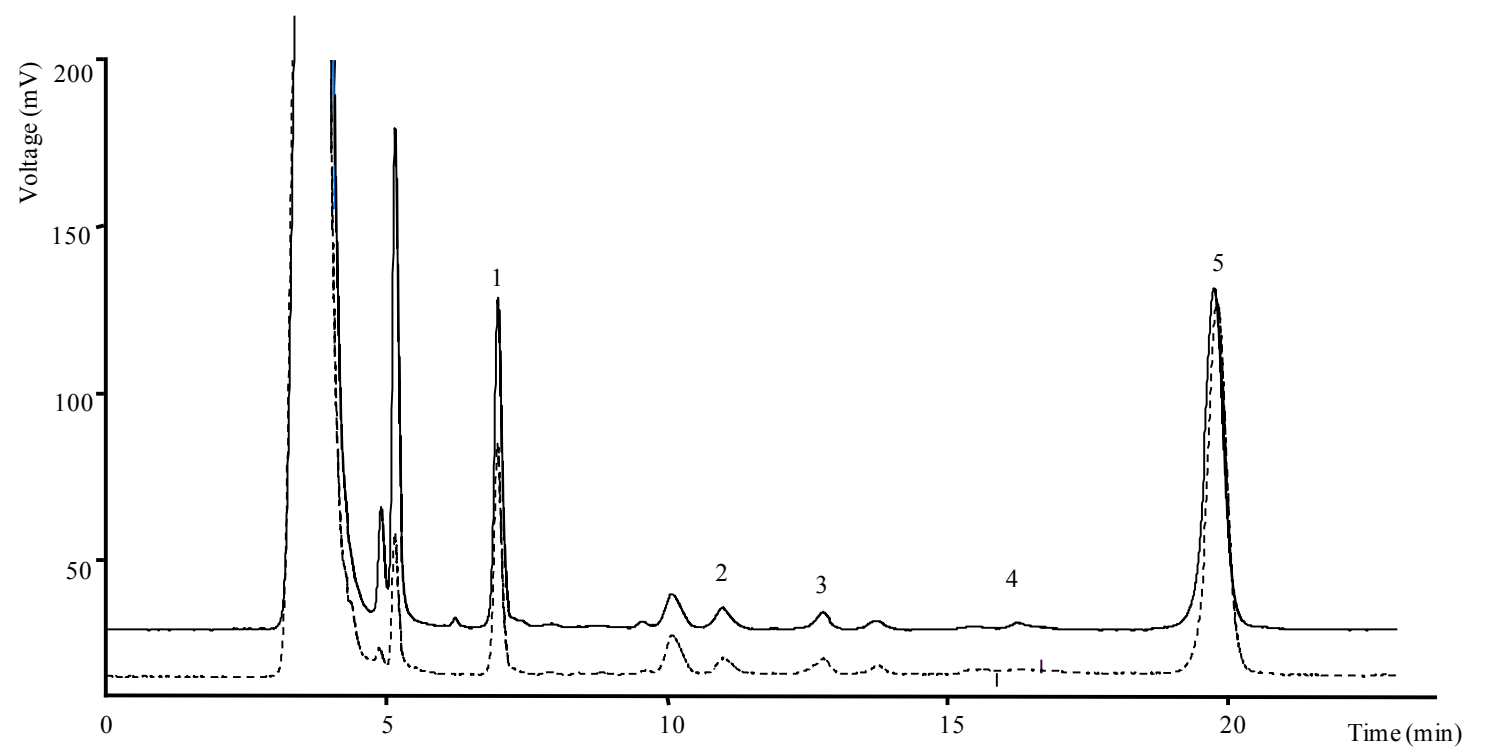

(A)

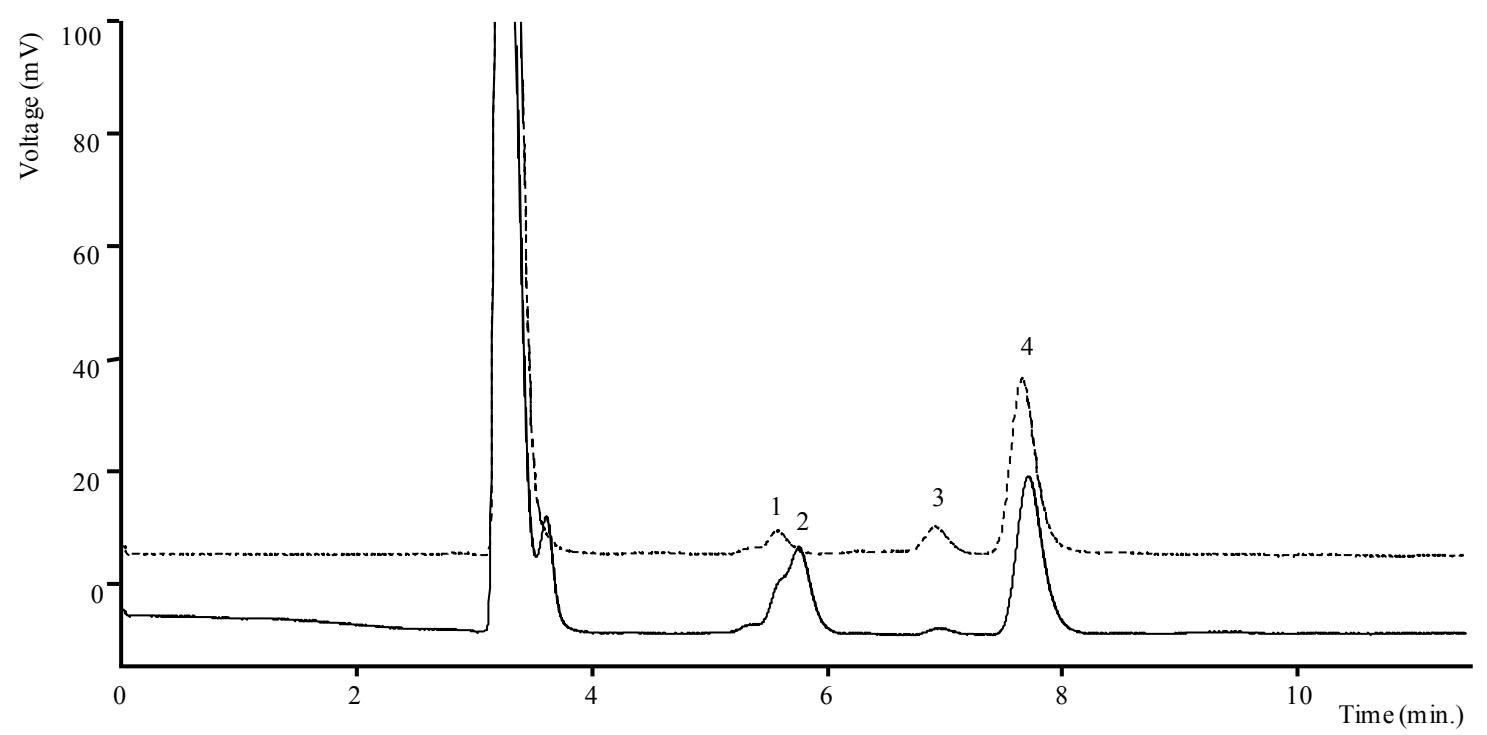

(B)

Figure 2. (A) Tocopherols profile of Castanea sativa roots in co-culture with Paxillus arhizus mycelium for $6 \mathrm{~h} \mathrm{(-)} \mathrm{and} 72 \mathrm{~h}(--) ; 1-\alpha$-tocopherol; 2 - BHT; 3 - $\beta$-tocopherol; 4$\gamma$-tocopherol; 5- $\delta$-tocopherol; 6- tocol (IS). (B) Sugars profile of Paxillus involutus (-) and Pisolithus arhizus (--) mycelium in co-culture with Catanea sativa root for $6 \mathrm{~h}$; 1glucose; 2- mannitol; 3- trehalose; 4- raffinose (IS). 


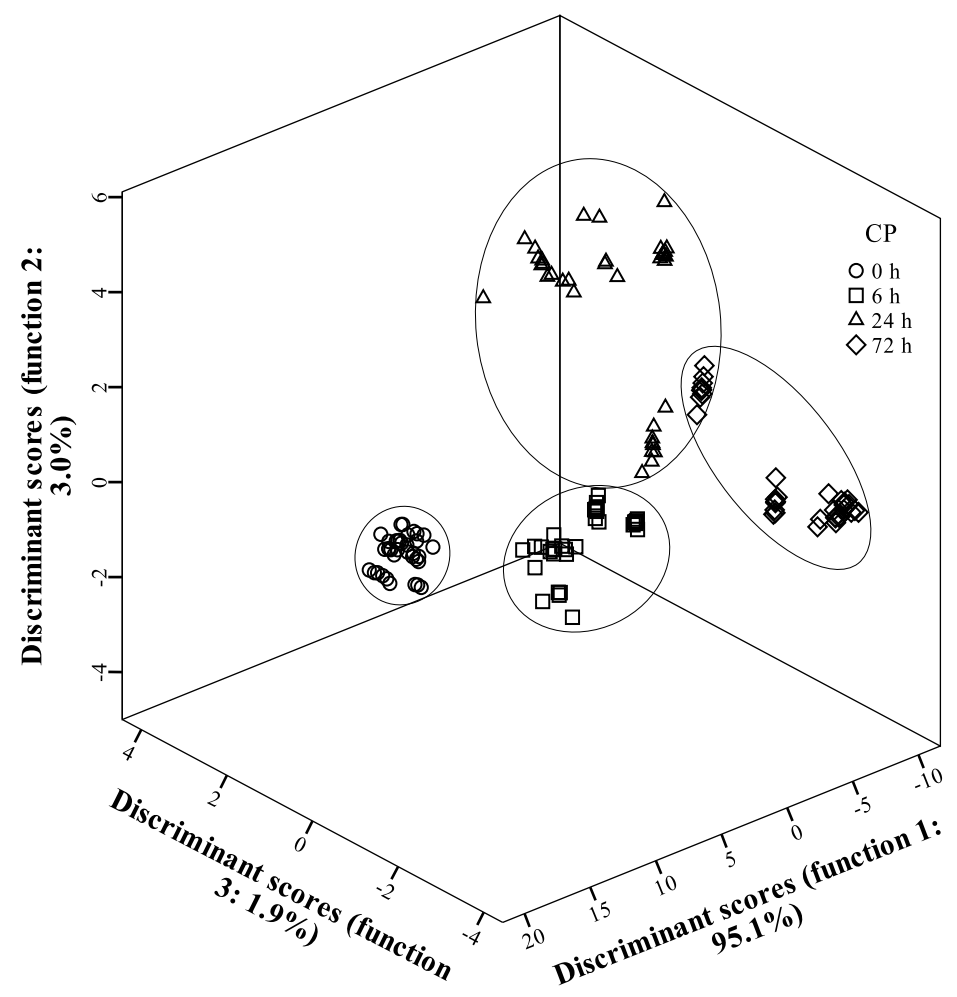

(A)

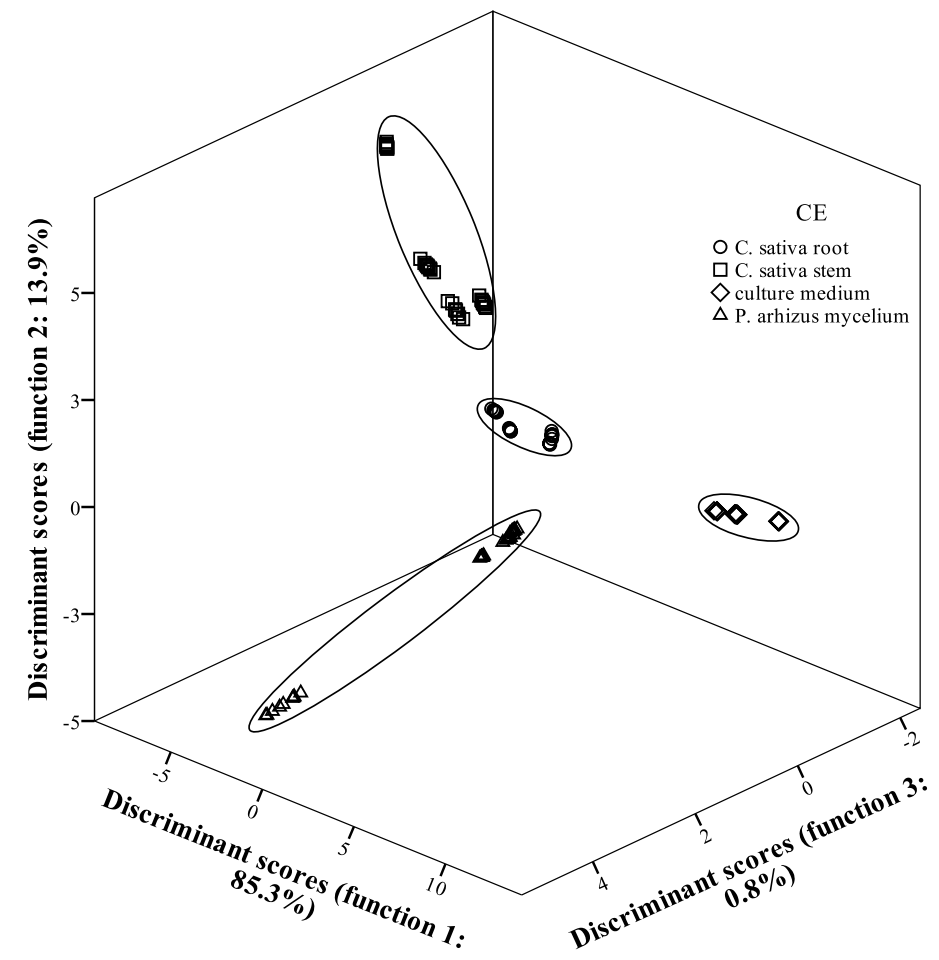

(B)

Figure 3. Canonical analysis of $P$. arhizus-C. sativa co-culture (A: separated by coculture period; B: separated by co-culture element) based on all the assayed parameters (A) or sugar contents (B). 


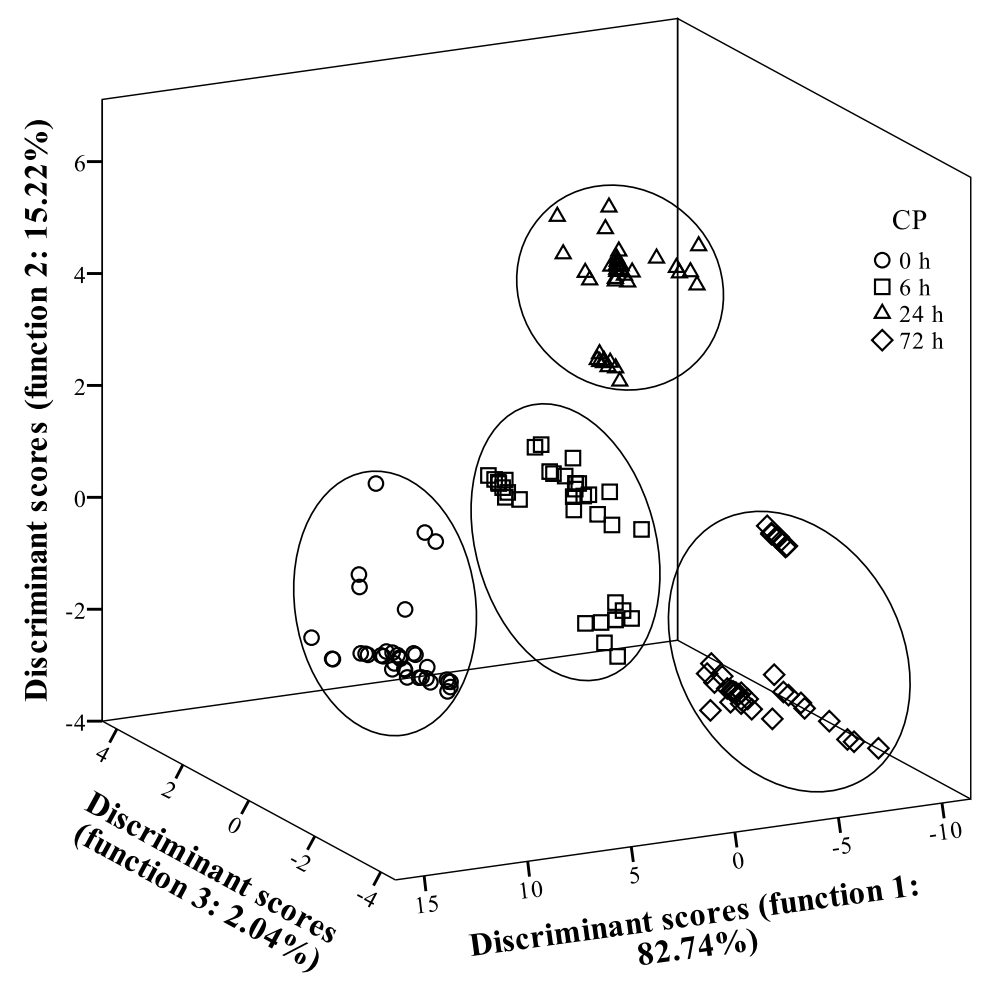

(A)

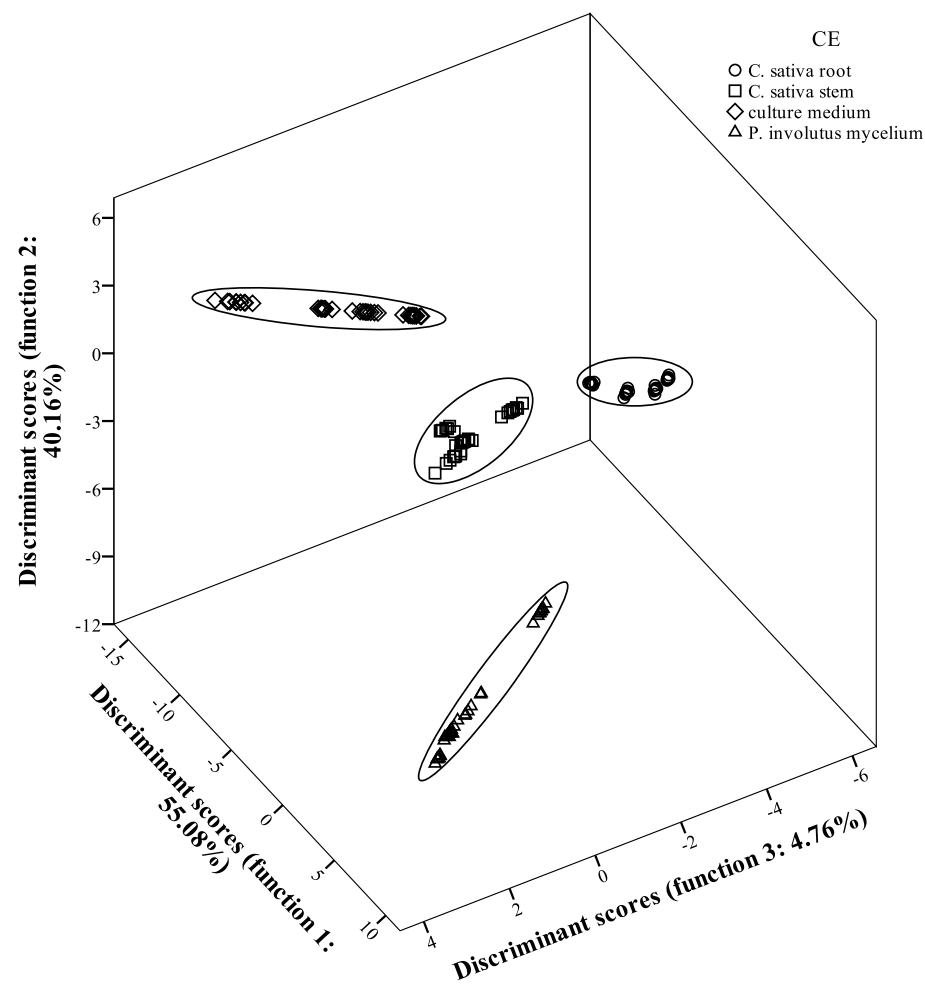

(B)

Figure 4. Canonical analysis of $P$. involutus-C. sativa co-culture (A: separated by coculture period; B: separated by co-culture element) based on all the assayed parameters (A) or sugar contents (B). 